\title{
LIGHTLIKE SURFACES WITH PLANAR NORMAL SECTIONS IN MINKOWSKI 3-SPACE
}

\author{
FEYZA ESRA ERDOĞAN, BAYRAM ŞAHIN, AND RIFAT GÜNEŞ
}

Dedicated to memory of Proffessor Franki Dillen

\begin{abstract}
In this paper we study lightlike surfaces of Minkowski 3- space such that they have degenerate or non-degenerate planar normal sections. We first show that every lightlike surface of Minkowski 3- space has degenerate planar normal sections. Then we study lightlike surfaces with non-degenerate planar normal sections and obtain a characterization for such lightlike surfaces
\end{abstract}

\section{INTRODUCTION}

Surfaces with planar normal sections in Euclidean spaces were first studied by Bang-Yen Chen [2]. Later such surfaces or submanifolds have been studied by many authors [2], [6], [7], [9],[8]. In [7], Y. H. Kim initiated the study of semi-Riemannian setting of such surfaces. But as far as we know, lightlike surfaces with planar normal sections have not been studied so far. Therefore, in this paper we study lightlike surfaces with planar normal sections of $\mathbb{R}_{1}^{3}$.

We first define the notion of surfaces with planar normal sections as follows. Let $M$ be a lightlike surface of $\mathbb{R}_{1}^{3}$. For a point $p$ in $M$ and a lightlike vector $\xi$ which spans the radical distribution of a lightlike surface, the vector $\xi$ and transversal space $\operatorname{tr}(T M)$ to $M$ at $p$ determine a 2- dimensional subspace $E(p, \xi)$ in $\mathbb{R}_{1}^{3}$ through $p$. The intersection of $M$ and $E(p, \xi)$ gives a lightlike curve $\gamma$ in a neighborhood of $p$, which is called the normal section of $M$ at the point $p$ in the direction of $\xi$.

For non-degenerate planar normal sections, we present the following notion. Let $w$ be a spacelike vector tangent to $M$ at $p$ which spans the chosen screen distribution of $M$. Then the vector $w$ and transversal space $\operatorname{tr}(T M)$ to $M$ at $p$ determine a 2dimensional subspace $E(p, w)$ in $\mathbb{R}_{1}^{3}$ through $p$. The intersection of $M$ and $E(p, w)$ gives a spacelike curve $\gamma$ in a neighborhood of $p$ which is called the normal section of $M$ at $p$ in the direction of $w$. According to both identifications above, $M$ is said to have degenerate pointwise and spacelike pointwise planar normal sections,

2010 Mathematics Subject Classification. 53C42, 53C50.

Key words and phrases. Lightlike surfaces non-degenerate planar normal section, degenerate normal section. 
respectively if each normal section $\gamma$ at $p$ satisfies $\gamma^{\prime} \wedge \gamma^{\prime \prime} \wedge \gamma^{\prime \prime \prime}=0$ at for each $p$ in $M$.

For a lightlike surface with degenerate planar normal sections, in fact, we show that every lightlike surface of Minkowski 3- space has degenerate planar normal sections. Then for a lightlike surface with non-degenerate planar normal sections, we obtain two characterizations.

We first show that a lightlike surface $M$ in $\mathbb{R}_{1}^{3}$ is a lightlike surface with nondegenerate planar sections if and only if $M$ is either screen conformal and totally umbilical or $M$ is totally geodesic. We also obtain a characterization for nonumbilical screen conformal lightlike surface with non-degenerate planar normal sections.

\section{Preliminaries}

Let $(\bar{M}, \bar{g})$ be an $(m+2)$-dimensional semi-Riemannian manifold with the indefinite metric $\bar{g}$ of index $q \in\{1, \ldots, m+1\}$ and $M$ be a hypersurface of $\bar{M}$. We denote the tangent space at $x \in M$ by $T_{x} M$. Then

$$
T_{x} M^{\perp}=\left\{V_{x} \in T_{x} \bar{M} \mid \bar{g}_{x}\left(V_{x}, W_{x}\right)=0, \forall W_{x} \in T_{x} M\right\}
$$

and

$$
\operatorname{Rad}_{x} M=T_{x} M \cap T_{x} M^{\perp} .
$$

Then, $M$ is called a lightlike hypersurface of $\bar{M}$ if $\operatorname{Rad} T_{x} M \neq\{0\}$ for any $x \in M$. Thus $T M^{\perp}=\bigcap_{x \in M} T_{x} M^{\perp}$ becomes a one- dimensional distribution $\operatorname{RadTM}$ on $M$. Then there exists a vector field $\xi \neq 0$ on $M$ such that

$$
g(\xi, X)=0, \quad \forall X \in \Gamma(T M),
$$

where $g$ is the induced degenerate metric tensor on $M$. We denote $F(M)$ the algebra of differential functions on $M$ and by $\Gamma(E)$ the $F(M)$ - module of differentiable sections of a vector bundle $E$ over $M$.

A complementary vector bundle $S(T M)$ of $T M^{\perp}=\operatorname{RadTM}$ in $T M$ i,e.,

$$
T M=\operatorname{Rad} T M \oplus_{\text {orth }} S(T M)
$$

is called a screen distribution on $M$. It follows from the equation above that $S(T M)$ is a non-degenerate distribution. Moreover, since we assume that $M$ is paracompact, there always exists a screen $S(T M)$. Thus, along $M$ we have the decomposition

$$
T \bar{M}_{\mid M}=S(T M) \oplus_{\text {orth }} S(T M)^{\perp}, \quad S(T M) \cap S(T M)^{\perp} \neq\{0\},
$$

that is, $S(T M)^{\perp}$ is the orthogonal complement to $S(T M)$ in $\left.T \bar{M}\right|_{M}$. Note that $S(T M)^{\perp}$ is also a non-degenerate vector bundle of rank 2 . However, it includes $T M^{\perp}=\operatorname{Rad} T M$ as its sub-bundle.

Let $(M, g, S(T M))$ be a lightlike hypersurface of a semi-Riemannian manifold $(\bar{M}, \bar{g})$. Then there exists a unique vector bundle $\operatorname{tr}(T M)$ of rank 1 over $M$, such that for any non-zero section $\xi$ of $T M^{\perp}$ on a coordinate neighborhood $U \subset M$, there exists a unique section $N$ of $\operatorname{tr}(T M)$ on $U$ satisfying: $T M^{\perp}$ in $S(T M)^{\perp}$ and 
take $V \in \Gamma\left(\left.F\right|_{U}\right), V \neq 0$. Then $\bar{g}(\xi, V) \neq 0$ on $U$, otherwise $S(T M)^{\perp}$ would be degenerate at a point of $U[5]$. Define a vector field

$$
N=\frac{1}{\bar{g}(V, \xi)}\left\{V-\frac{\bar{g}(V, V)}{2 \bar{g}(V, \xi)} \xi\right\}
$$

on $U$ where $V \in \Gamma\left(\left.F\right|_{U}\right)$ such that $\bar{g}(\xi, V) \neq 0$. Then we have

$$
\bar{g}(N, \xi)=1, \bar{g}(N, N)=0, \bar{g}(N, W)=0, \forall W \in \Gamma\left(\left.S(T M)\right|_{U}\right)
$$

Moreover, from (2.1) and (2.2) we have the following decompositions:

$$
\left.T \bar{M}\right|_{M}=S(T M) \oplus_{\text {orth }}\left(T M^{\perp} \oplus \operatorname{tr}(T M)\right)=T M \oplus \operatorname{tr}(T M)
$$

Locally, suppose $\{\xi, N\}$ is a pair of sections on $U \subset M$ satisfying (2.3). Define a symmetric $\Im(U)$-bilinear from $B$ and a 1 -form $\tau$ on $U$. Hence on $U$, for $X, Y \in$ $\Gamma\left(\left.T M\right|_{U}\right)$

$$
\begin{aligned}
\bar{\nabla}_{X} Y & =\nabla_{X} Y+B(X, Y) N \\
\bar{\nabla}_{X} N & =-A_{N} X+\tau(X) N,
\end{aligned}
$$

equations (2.5) and (2.6) are local Gauss and Weingarten formulae. Since $\bar{\nabla}$ is a metric connection on $\bar{M}$, it is easy to see that

$$
B(X, \xi)=0, \forall X \in \Gamma\left(\left.T M\right|_{U}\right) .
$$

Consequently, the second fundamental form of $M$ is degenerate [5]. Define a local 1-from $\eta$ by

$$
\eta(X)=\bar{g}(X, N), \forall \in \Gamma\left(\left.T M\right|_{U}\right)
$$

Let $P$ denote the projection morphism of $\Gamma(T M)$ on $\Gamma(S(T M))$ with respect to the decomposition (2.1). We obtain

$$
\begin{aligned}
\nabla_{X} P Y & =\nabla_{X}^{*} P Y+C(X, P Y) \xi \\
\nabla_{X} \xi & =-A_{\xi}^{*} X+\varepsilon(X) \xi \\
& =-A_{\xi}^{*} X-\tau(X) \xi
\end{aligned}
$$

where $\nabla_{X}^{*} Y$ and $A_{\xi}^{*} X$ belong to $\Gamma(S(T M)), \nabla$ and $\nabla^{* t}$ are linear connections on $\Gamma(S(T M))$ and $T M^{\perp}$ respectively, $h^{*}$ is a $\Gamma\left(T M^{\perp}\right)$-valued $\Im(M)$-bilinear form on $\Gamma(T M) \times \Gamma(S(T M))$ and $A_{\xi}^{*}$ is $\Gamma(S(T M))$-valued $\Im(M)$-linear operator on $\Gamma(T M)$. We called them the screen fundamental form and screen shape operator of $S(T M)$, respectively. Define

$$
\begin{aligned}
C(X, P Y) & =\bar{g}\left(h^{*}(X, P Y), N\right) \\
\varepsilon(X) & =\bar{g}\left(\nabla_{X}^{* t} \xi, N\right), \forall X, Y \in \Gamma(T M),
\end{aligned}
$$

one can show that $\varepsilon(X)=-\tau(X)$. Here $C(X, P Y)$ is called the local screen fundamental form of $S(T M)$. Precisely, the two local second fundamental forms of $M$ and $S(T M)$ are related to their shape operators by

$$
\begin{aligned}
B(X, Y) & =\bar{g}\left(Y, A_{\xi}^{*} X\right), \\
A_{\xi}^{*} \xi & =0 \\
\bar{g}\left(A_{\xi}^{*} P Y, N\right) & =0 \\
C(X, P Y) & =\bar{g}\left(P Y, A_{N} X\right), \\
\bar{g}\left(N, A_{N} X\right) & =0 .
\end{aligned}
$$


A lightlike hypersurface $(M, g, S(T M))$ of a semi-Riemannian manifold is called totally umbilical[5] if there is a smooth function $\varrho$, such that

$$
B(X, Y)=\varrho g(X, Y), \forall X, Y \in \Gamma(T M)
$$

where $\varrho$ is non-vanishing smooth function on a neighborhood $U$ in $M$.

A lightlike hypersurface $(M, g, S(T M))$ of a semi-Riemannian manifold is called screen locally conformal if the shape operators $A_{N}$ and $A_{\xi}^{*}$ of $M$ and $S(T M)$, respectively, are related by

$$
A_{N}=\varphi A_{\xi}^{*}
$$

where $\varphi$ is non-vanishing smooth function on a neighborhood $U$ in $M$. Therefore, it follows that $\forall X, Y \in \Gamma(S(T M)), \xi \in \operatorname{RadTM}$

$$
C(X, \xi)=0,
$$

For details about screen conformal lightlike hypersurfaces, see: [1] and [5] .

\section{Planar normal sections of lightlike surfaces in $\mathbb{R}_{1}^{3}$}

Let $M$ be a lightlike surface of $\mathbb{R}_{1}^{3}$. Now we investigate lightlike surfaces with degenerate planar normal sections. If $\gamma$ is a null curve, for a point $p$ in $M$, we have

$$
\begin{aligned}
\gamma^{\prime}(s) & =\xi \\
\gamma^{\prime \prime}(s) & =\bar{\nabla}_{\xi} \xi=-\tau(\xi) \xi \\
\gamma^{\prime \prime \prime}(s) & =-\left[\xi(\tau(\xi))+\tau^{2}(\xi)\right] \xi
\end{aligned}
$$

Then, $\gamma^{\prime \prime \prime}(0)$ is a linear combination of $\gamma^{\prime}(0)$ and $\gamma^{\prime \prime}(0)$. Thus (3.1), (3.2) and (3.3) give $\gamma^{\prime \prime \prime}(0) \wedge \gamma^{\prime \prime}(0) \wedge \gamma^{\prime}(0)=0$. Thus lightlike surfaces always have planar normal sections.

Corollary 3.1. Every lightlike surface of $\mathbb{R}_{1}^{3}$ has degenerate planar normal sections.

In fact Corollary 3.1 tells us that the above situation is not interesting. Now, we will check lightlike surfaces with non-degenerate planar normal sections. Let $M$ be a lightlike hypersurface of $\mathbb{R}_{1}^{3}$. For a point $p$ in $M$ and a spacelike vector $w \in S(T M)$ tangent to $M$ at $p$, the vector $w$ and transversal space $\operatorname{tr}(T M)$ to $M$ at $p$ determine a 2-dimensional subspace $E(p, w)$ in $\mathbb{R}_{1}^{3}$ through $p$. The intersection of $M$ and $E(p, w)$ give a spacelike curve $\gamma$ in a neighborhood of $p$, which is called the normal section of $M$ at $p$ in the direction of $w$. Now, we research the conditions for a lightlike surface of $\mathbb{R}_{1}^{3}$ to have non-degenerate planar normal sections.

Let $(M, g, S(T M))$ be a screen conformal lightlike surface of $\left(\bar{g}, \mathbb{R}_{1}^{3}\right)$. In this case $S(T M)$ is integrable[1]. We denote integral submanifold of $S(T M)$ by $M^{\prime}$. Then, using (2.6), (2.10) and (2.19) we obtain

(3.4) $\gamma^{\prime}(s)=w$

(3.5) $\gamma^{\prime \prime}(s)=\bar{\nabla}_{w} w=\nabla_{w}^{*} w+C(w, w) \xi+B(w, w) N$

(3.6) $\gamma^{\prime \prime \prime}(s)=\nabla_{w}^{*} \nabla_{w}^{*} w+C\left(w, \nabla_{w}^{*} w\right) \xi+w(C(w, w)) \xi-C(w, w) A_{\xi}^{*} w$ $+w(B(w, w)) N-B(w, w) A_{N} w+B\left(w, \nabla_{w}^{*} w\right) N$ 
Where $\nabla^{*}$ and $\nabla$ are linear connections on $S(T M)$ and $\Gamma(T M)$, respectively and $\gamma^{\prime}(s)=w$. From the definition of planar normal section and that $S(T M)=$ $S p\{w\}$, we have

$$
w \wedge \nabla_{w}^{*} w=0
$$

and

$$
w \wedge \nabla_{w}^{*} \nabla_{w}^{*} w=0 .
$$

Then, from (3.5), (3.6) and (3.7), (3.8) we obtain $\gamma^{\prime \prime \prime}(s) \wedge \gamma^{\prime \prime}(s) \wedge \gamma^{\prime}(s)=0$. Thus, $M$ has planar non-degenerate normal sections.

If $M$ is totally geodesic lightlike surface of $\mathbb{R}_{1}^{3}$. Then, we have $B=0, A_{\xi}^{*}=0$. Hence (3.4)-(3.6) become

$$
\begin{aligned}
\gamma^{\prime}(s) & =w \\
\gamma^{\prime \prime}(s) & =\nabla_{w}^{*} w+C(w, w) \xi \\
\gamma^{\prime \prime \prime}(s) & =\nabla_{w}^{*} \nabla_{w}^{*} w+t C(w, w) \xi+w(C(w, w)) \xi
\end{aligned}
$$

where $\nabla_{w}^{*} w=t w, t \in \mathbb{R}$. Since $A_{N} w \in \Gamma(T M)$, we have $\gamma^{\prime \prime \prime}(s) \wedge \gamma^{\prime \prime}(s) \wedge \gamma^{\prime}(s)=0$.

Conversely, we assume that $M$ has planar non-degenerate normal sections. Then, from (3.4), (3.5), (3.6) and (3.7), (3.8) we obtain

$$
(C(w, w) \xi+B(w, w) N) \wedge\left(C(w, w) A_{\xi}^{*} w+B(w, w) A_{N} w\right)=0,
$$

thus $\left(C(w, w) A_{\xi}^{*} w+B(w, w) A_{N} w\right)=0$ or $C(w, w) \xi+B(w, w) N=0$. If $C(w, w) A_{\xi}^{*} w+B(w, w) A_{N} w=0$, then, from

$$
A_{\xi}^{*} w=-\frac{B(w, w)}{C(w, w)} A_{N} w
$$

at $p \in M, M$ is a screen conformal lightlike surface with $C(w, w) \neq 0$. If $C(w, w) \xi+$ $B(w, w) N=0$, then $\operatorname{RadTM}$ is parallel and $M$ is totally geodesic.

Consequently, we have the following,

Theorem 3.1. Let $M$ be a lightlike surface of $\mathbb{R}_{1}^{3}$. Then $M$ has non-degenerate planar normal sections if and only if either $M$ is screen conformal or $M$ is totally geodesic.

Theorem 3.2. Let $(M, g, S(T M))$ be a screen conformal non-umbilical lightlike surface of $\mathbb{R}_{1}^{3}$. Then, for $T(w, w)=C(w, w) \xi+B(w, w) N$ the following statements are equivalent

(1) $\left(\bar{\nabla}_{w} T\right)(w, w)=0$, every spacelike vector $w \in S(T M)$

(2) $\bar{\nabla} T=0$

(3) $M$ has non-degenerate planar normal sections and each normal section at $p$ has one of its vertices at $p$

By the vertex of curve $\gamma(s)$ we mean a point $p$ on $\gamma$ such that its curvature $\kappa$ satisfies $\frac{d \kappa^{2}(p)}{d s}=0, \kappa^{2}=\left\langle\gamma^{\prime \prime}(s), \gamma^{\prime \prime}(s)\right\rangle$. 
Proof. From (3.4), (3.5) and that a screen conformal $M$, we have

$$
\left(\bar{\nabla}_{w} T\right)(w, w)=\bar{\nabla}_{w} T(w, w)
$$

which shows $(a) \Leftrightarrow(b) . \quad(b) \Rightarrow(c)$ Assume that $\bar{\nabla} T=0$. If $\bar{\nabla} T=0$ then $M$ is totally geodesic and Theorem 3.1 implies that $M$ has (pointwise) planar normal sections. Let the $\gamma(s)$ be a normal section of $M$ at $p$ in a given direction $w \in S(T M)$. Then (3.4) shows that the curvature $\kappa(s)$ of $\gamma(s)$ satisfies

$$
\begin{aligned}
\kappa^{2}(s) & =\left\langle\gamma^{\prime \prime}(s), \gamma^{\prime \prime}(s)\right\rangle \\
& =2 C(w, w) B(w, w) \\
& =\langle T(w, w), T(w, w)\rangle
\end{aligned}
$$

where $w=\gamma^{\prime}(s)$. Therefore we find

$$
\frac{d \kappa^{2}(p)}{d s}=\left\langle\bar{\nabla}_{w} T(w, w), T(w, w)\right\rangle=\left\langle\left(\bar{\nabla}_{w} T\right)(w, w), T(w, w)\right\rangle
$$

Since $\bar{\nabla}_{w} T(w, w)=0$, this implies

$$
\frac{d \kappa^{2}(0)}{d s}=0
$$

at $p=\gamma(0)$. Thus $p$ is a vertex of the normal section $\gamma(s)$. $(c) \Rightarrow(a)$ : If $M$ has planar normal sections, then Theorem 3.1 gives

$$
T(w, w) \wedge\left(\bar{\nabla}_{w} T\right)(w, w)=0 .
$$

If $p$ is a vertex of $\gamma(s)$, then we have

$$
\frac{d \kappa^{2}(0)}{d s}=0
$$

Thus, since $M$ has planar normal sections using (3.10) we find

$$
\begin{aligned}
\gamma^{\prime}(s) \wedge \gamma^{\prime \prime}(s) \wedge \gamma^{\prime \prime \prime}(s)= & w \wedge\left(\nabla_{w}^{*} w+T(w, w)\right) \\
& \wedge\left(\nabla_{w}^{*} \nabla_{w}^{*} w+t T(w, w)+\left(\bar{\nabla}_{w} T\right)(w, w)\right)=0 \\
\gamma^{\prime}(s) \wedge \gamma^{\prime \prime}(s) \wedge \gamma^{\prime \prime \prime}(s)= & T(w, w) \wedge\left(\bar{\nabla}_{w} T\right)(w, w)=0
\end{aligned}
$$

and

$$
\left\langle\left(\bar{\nabla}_{w} T\right)(w, w), T(w, w)\right\rangle=0 .
$$

Combining (3.11) and (3.12) we obtain $\left(\bar{\nabla}_{w} T\right)(w, w)=0$ or $T(w, w)=0$. Let us define $U=\{w \in S(T M) \mid T(w, w)=0\}$. If $\operatorname{int}(U) \neq \emptyset$, we obtain $\left(\bar{\nabla}_{w} T\right)(w, w)=$ 0 on $\operatorname{int}(U)$. Thus, by continuity we have $\bar{\nabla} T=0$.

Example 3.1. Consider the null cone of $\mathbb{R}_{1}^{3}$ given by

$$
\wedge=\left\{\left(x_{1}, x_{2}, x_{3}\right) \mid-x_{1}^{2}+x_{2}^{2}+x_{3}^{2}=0, x_{1}, x_{2}, x_{3} \in I R\right\} .
$$

The radical bundle of null cone is

$$
\xi=x_{1} \frac{\partial}{\partial x_{1}}+x_{2} \frac{\partial}{\partial x_{2}}+x_{3} \frac{\partial}{\partial x_{3}}
$$

and screen distribution is spanned by

$$
Z_{1}=x_{2} \frac{\partial}{\partial x_{1}}+x_{3} \frac{\partial}{\partial x_{2}}
$$


Then the lightlike transversal vector bundle is given by

$$
\operatorname{Itr}(T M)=\operatorname{Span}\left\{N=\frac{1}{2\left(-x_{1}^{2}+x_{2}^{2}\right)}\left(x_{1} \frac{\partial}{\partial x_{1}}+x_{2} \frac{\partial}{\partial x_{2}}-x_{3} \frac{\partial}{\partial x_{3}}\right)\right\} .
$$

It follows that the corresponding screen distribution $S(T M)$ is spanned by $Z_{1}$. Thus

$$
\begin{aligned}
\nabla_{\xi} \xi & =x_{1} \frac{\partial}{\partial x_{1}}+x_{2} \frac{\partial}{\partial x_{2}}+x_{3} \frac{\partial}{\partial x_{3}} \\
\bar{\nabla}_{\xi} \nabla_{\xi} \xi & =x_{1} \frac{\partial}{\partial x_{1}}+x_{2} \frac{\partial}{\partial x_{2}}+x_{3} \frac{\partial}{\partial x_{3}} .
\end{aligned}
$$

Then, we obtain

$$
\gamma^{\prime \prime \prime}(s) \wedge \gamma^{\prime \prime}(s) \wedge \gamma^{\prime}(s)=0
$$

which shows that null cone has degenerate planar normal sections.

Example 3.2. Let $\mathbb{R}_{1}^{3}$ be the space $\mathbb{R}_{1}^{3}$ endowed with the semi Euclidean metric

$$
\bar{g}(x, y)=-x_{1} y_{1}+x_{2} y_{2}+x_{3} y_{3},\left(x=\left(x_{1}, x_{2}, x_{3}\right)\right) .
$$

The lightlike cone $\wedge_{0}^{2}$ is given by the equation $-\left(x_{1}\right)^{2}+\left(x_{2}\right)^{2}+\left(x_{3}\right)^{2}=0, x \neq 0$. It is known that $\wedge_{0}^{2}$ is a lightlike surface of $\mathbb{R}_{1}^{3}$ and the radical distribution is spanned by a global vector field

$$
\xi=x_{1} \frac{\partial}{\partial x_{1}}+x_{2} \frac{\partial}{\partial x_{2}}+x_{3} \frac{\partial}{\partial x_{3}}
$$

on $\wedge_{0}^{2}$. The unique section $N$ is given by

$$
N=\frac{1}{2\left(x_{1}\right)^{2}}\left(-x_{1} \frac{\partial}{\partial x_{1}}+x_{2} \frac{\partial}{\partial x_{2}}+x_{3} \frac{\partial}{\partial x_{3}}\right)
$$

and is also defined. As $\xi$ is the position vector field we get

$$
\bar{\nabla}_{X} \xi=\nabla_{X} \xi=X, \quad \forall X \in \Gamma(T M) .
$$

Then, $A_{\xi}^{*} X+\tau(X) \xi+X=0$. As $A_{\xi}^{*}$ is $\Gamma(S(T M))$-valued we obtain

$$
A_{\xi}^{*} X=-P X, \quad \forall X \in \Gamma(T M)
$$

Next, any $X \in \Gamma\left(S\left(T \wedge_{0}^{2}\right)\right)$ is expressed by $X=X_{2} \frac{\partial}{\partial x_{2}}+X_{3} \frac{\partial}{\partial x_{3}}$ where $\left(X_{2}, X_{3}\right)$ satisfy

$$
x_{2} X_{2}+x_{3} X_{3}=0
$$

and then

$$
\begin{aligned}
\nabla_{\xi} X & =\bar{\nabla}_{\xi} X=\sum_{A=1}^{3} \sum_{a=2}^{3} x_{A} \frac{\partial X_{a}}{\partial x_{A}} \frac{\partial}{\partial x_{a}} \\
\bar{g}\left(\nabla_{\xi} X, \xi\right) & =\sum_{A=1}^{3} \sum_{a=2}^{3} x_{a} x_{A} \frac{\partial X^{a}}{\partial x_{A}}=-\left(x_{2} X_{2}+x_{3} X_{3}\right)=0
\end{aligned}
$$

where (3.17) is derived with respect to $x_{1}, x_{2}, x_{3}$. It is known that $\wedge_{0}^{2}$ is a screen conformal lightlike surface with conformal function $\varphi=\frac{1}{2\left(x_{1}\right)^{2}}$. We also know that $A_{N} \xi=0$. By direct compute we find

$$
A_{N} X=\frac{1}{2\left(x_{1}\right)^{2}} A_{\xi}^{*} X
$$


Now we evaluate $\gamma^{\prime}, \gamma^{\prime \prime}$ and $\gamma^{\prime \prime \prime}$

$$
\begin{aligned}
\gamma^{\prime} & =X=\left(0,-x_{3}, x_{2}\right) \\
\gamma^{\prime \prime} & =\nabla_{X} X+B(X, X) N \\
& =\frac{1}{2} x_{1} \frac{\partial}{\partial x_{1}}-\frac{3}{2} x_{2} \frac{\partial}{\partial x_{2}}+x_{3} \frac{\partial}{\partial x_{3}} \\
\gamma^{\prime \prime \prime} & =\bar{\nabla}_{X} \nabla_{X} X+X(B(X, X)) N+B(X, X) \bar{\nabla}_{X} N \\
& =\nabla_{X} \nabla_{X} X+B\left(X, \nabla_{X} X\right) N+X(B(X, X)) N-B(X, X) A_{N} X
\end{aligned}
$$

using $A_{N} X$ in $\gamma^{\prime \prime \prime}$ we get

$$
\gamma^{\prime \prime \prime}=\frac{1}{2} x_{3} \frac{\partial}{\partial x_{2}}-\frac{1}{2} x_{2} \frac{\partial}{\partial x_{3}} .
$$

Therefore $\gamma^{\prime \prime \prime}$ and $\gamma^{\prime}$ are linear dependence at $\forall p \in \wedge_{0}^{2}$ and we have

$$
\gamma^{\prime} \wedge \gamma^{\prime \prime} \wedge \gamma^{\prime \prime \prime}=0 .
$$

Namely, $\wedge_{0}^{2}$ has non-degenerate planar normal sections.

Example 3.3. Consider the lightlike surface $M$ of $\mathbb{R}_{1}^{3}$ given by

$$
\left(x_{1}+1\right)=\sqrt{x_{2}^{2}+x_{3}^{2}}, x_{2} \neq x_{3} \neq 0 .
$$

Then its radical distributions spanned by

$$
\xi=\left(x_{1}+1\right) \partial x_{1}+x_{2} \partial x_{2}+x_{3} \partial x_{3}
$$

and the lightlike transversal vector bundle is spanned by

$$
N=-\frac{1}{2\left(x_{1}+1\right)^{2}}\left(\left(x_{1}+1\right) \partial x_{1}-x_{2} \partial x_{2}-x_{3} \partial x_{3}\right) .
$$

It follow that the corresponding screen distribution $S(T M)$ is spanned by

$$
w=-x_{3} \partial x_{2}+x_{2} \partial x_{3} .
$$

By direct computations, we obtain

$$
\bar{\nabla}_{\xi} w=\nabla_{\xi} w=\bar{\nabla}_{\xi} w=\nabla_{\xi} w=w
$$

and

$$
B(w, w)=-\bar{g}(w, w)=-\left(x_{1}+1\right)^{2} .
$$

Hence $M$ is totally umbilical lightlike surface and direct computations, we obtain

and

$$
\begin{gathered}
\bar{\nabla}_{w} w=\frac{1}{2}\left(x_{1}+1\right)-\frac{3}{2} x_{2} \partial x_{2}-\frac{3}{2} x_{3} \partial x_{3}, \\
C(w, w)=-\frac{1}{2}
\end{gathered}
$$

$$
A_{N} w=\frac{1}{2\left(x_{1}+1\right)^{2}} A_{\xi}^{*} w
$$

Hence $M$ is screen conformal lightlike surface. On the other hand, we have

$$
\begin{gathered}
\bar{\nabla}_{\xi} \nabla_{\xi} \xi=\bar{\nabla}_{\xi} \xi=\left(x_{1}+1\right) \partial x_{1}+x_{2} \partial x_{2}+x_{3} \partial x_{3}, \\
w(B(w, w))=B\left(w, \nabla_{w} w\right)=0, \\
A_{N} w=\frac{1}{2\left(x_{1}+1\right)^{2}}\left(x_{3} \partial x_{2}-x_{2} \partial x_{3}\right),
\end{gathered}
$$




$$
\bar{\nabla}_{w} \bar{\nabla}_{w} w=-\frac{2\left(x_{1}+1\right)^{2}-1}{2\left(x_{1}+1\right)^{2}} w .
$$

Now, for a point $p$ in $M$ and a spacelike vector $w$ tangent to $M$ at $p(w \in S(T M))$, the vector $w$ and transversal space $\operatorname{tr}(T M)$ to $M$ at $p$ determine an 2-dimensional subspace $E(p, w)$ in $\mathbb{R}_{1}^{3}$ through $p$. The intersection of $M$ and $E(p, w)$ gives a spacelike curve $\gamma$ in a neighborhood of $p$. Therefore, we have

$$
\begin{aligned}
\gamma^{\prime}(s) & =w=-x_{3} \partial x_{2}+x_{2} \partial x_{3} \\
\gamma^{\prime \prime}(s) & =\bar{\nabla}_{w} w=\frac{1}{2}\left(x_{1}+1\right)-\frac{3}{2} x_{2} \partial x_{2}-\frac{3}{2} x_{3} \partial x_{3} \\
\gamma^{\prime \prime \prime}(s) & =-\frac{2\left(x_{1}+1\right)^{2}-1}{2\left(x_{1}+1\right)^{2}}\left(-x_{3} \partial x_{2}+x_{2} \partial x_{3}\right) .
\end{aligned}
$$

Therefore $\gamma^{\prime \prime \prime}$ and $\gamma^{\prime}$ are linear dependence at $\forall p \in M$ and we have

$$
\gamma^{\prime} \wedge \gamma^{\prime \prime} \wedge \gamma^{\prime \prime \prime}=0
$$

Namely, $M$ has non-degenerate planar normal sections. Now, for a point $p$ in $M$ and a lightlike vector $\xi$ tangent to $M$ at $p(\xi \in \operatorname{Rad}(T M))$, the vector $\xi$ and transversal space $\operatorname{tr}(T M)$ to $M$ at $p$ determine an 2-dimensional subspace $E(p, \xi)$ in $\mathbb{R}_{1}^{3}$ through $p$. The intersection of $M$ and $E(p, \xi)$ gives a null curve $\gamma$ in a neighborhood of $p$. Then, we have

$$
\gamma^{\prime}(s)=\gamma^{\prime \prime}(s)=\gamma^{\prime \prime \prime}(s)=\xi
$$

Therefore $\gamma^{\prime \prime \prime}, \gamma^{\prime \prime}$ and $\gamma^{\prime}$ are linear dependence at $\forall p \in M$. Namely, M has degenerate planar normal sections.

\section{REFERENCES}

[1] Atindogbe, C. Duggal, K.L.,Conformal screen on lightlike hypersurfaces, Int. J. Pure Appl. Math. 11(4), (2004), 421-442.

[2] Chen, B.Y.,Classification of Surfaces with Planar Normal Sections, Journal of Geometry Vol.20 (1983) 122-127

[3] Duggal, K. L. and Bejancu, A., Lightlike Submanifolds of Semi-Riemannian Manifolds and Applications, Kluwer Academic Publisher, 1996.

[4] Duggal, K. L. and Jin, D. H., Null Curves and Hypersurfaces of Semi Riemannian Manifolds, World Scientific, 2007.

[5] Duggal, K. L. and Sahin B., Differential Geometry of Lightlike Submanifolds, Springer Birkhauser,2010.

[6] Kim, Y. H.,Minimal surfaces of pseudo-Euclidean spaces with geodesic normal sections, Differential Geometry and its Applications 5 (1995) 321-329.

[7] Kim, Y. H.,Pseudo-Riemannian Submanifolds with pointwise planar normal sections, Math. J. Okayama Univ.34 (1992), 249-257.

[8] Kim, Y. H.,Surfaces in a pseudo-Euclidean space with planar normal sections, Journal of Geometry Vol.35 (1989),120-131.

[9] Li. S. J.,Submanifolds with Pointwise Planar Normal Sections in a sphere, Journal of Geometry, 70 (2001) 101-107.

[10] O'Neill , B., Semi Riemannian Geometry with Applications to Relativity, Academic Press, 1983. 
Faculty of Arts and Science, Department of Mathematics, Adiyaman University, 02040 ADIYAMAN, TURKEY

E-mail address: ferdogan@adiyaman.edu.tr

Department of Mathematics, İNÖnÜ University, 44280 Malatya, TURKEY

E-mail address: bayram.sahin@inonu.edu.tr

Department of Mathematics, İnÖnÜ University, 44280 Malatya, TURKEY

E-mail address: rifat.gunes@inonu.edu.tr 